\title{
Ocular and Systemic Complications of COVID-19: Impact on Patients and Healthcare
}

\author{
Ella H Leung' \\ Jason Fan (D) ${ }^{2}$ \\ Harry W Flynn Jr (D) ${ }^{2}$ \\ Thomas A Albini² \\ 'Georgia Retina, Atlanta, GA, USA; \\ ${ }^{2}$ Bascom Palmer Eye Institute/University \\ of Miami, Miami, FL, USA
}

\begin{abstract}
There is increasing information available about the effects of the SARS-CoV-2 virus on the systemic and ocular health of patients, as well as the effects of delayed health care. This mini-review summarizes the potential complications and treatments of COVID-19. Systemic findings include respiratory illness, risk of thromboembolic events, and neurologic findings. Some patients may develop persistent symptoms even after the infection resolves. Effective treatment options include glucocorticoids, antivirals, interleukin-6 antagonists, monoclonal antibodies, Janus kinase inhibitors and vaccines. Potential ocular findings of COVID-19 include conjunctivitis, cranial nerve palsies, and microvascular changes in the retina; most symptoms resolved over time. During the lockdown periods, teleophthalmology was utilized to triage non-urgent issues; patients who did present to emergency departments tended to have more severe disease with worse visual prognoses. While transient delays in outpatient ophthalmic care may be tolerated in some patients, others experienced significant vision loss with interruptions in treatments. Resumption of ophthalmic care as soon as possible may help mitigate the effects of delayed care due to the pandemic.
\end{abstract}

Keywords: COVID-19, SARS-CoV-2, quarantine, steroids, interleukin 6, tocilizumab, monoclonal antibodies, casirivimab, imdevimab, antiviral, remdesivir; teleophthalmology; vaccine; trauma

\section{Introduction}

What began as an infection in China in 2019 continues as a global pandemic into 2022. COVID-19 is characterized by fever and severe respiratory illness or pneumonia, but it can also affect the ophthalmic, neurological, cardiovascular, gastroenterological, and nephrological systems. ${ }^{1,2}$ As the severe acute respiratory syndrome coronavirus 2 (SARS-CoV-2) virus continues to mutate, the farreaching effects of COVID-19 will likely persist. According to the World Health Organization, over 276 million people worldwide have had confirmed COVID-19 infection as of December 2021, and over 5 million people having died from the disease and its complications. ${ }^{3}$ Public health measures to curtail the pandemic include social distancing, wearing masks, and vaccinating the population. ${ }^{4}$

This mini-review seeks to summarize the current information on the complications of the COVID-19 pandemic on the systemic and ocular health of patients, review current treatment regimens shown to be efficacious in large meta-analyses and systemic reviews, evaluate the effects of delays in healthcare, review the efficacy and potential side effects of vaccines, and review the impact of the pandemic on ophthalmic practices, education, and research.
Correspondence: Ella H Leung Georgia Retina, 833 Campbell Hill St NW, Suite 300, Marietta, GA, 30328, USA

Tel +I 770-2I8-I888

Email eleung@garetina.com 


\section{Systemic Findings COVID-19 Transmission and Infection}

SARS-CoV-2 virus binds to the angiotensin converting enzyme (ACE2) receptors, which are present in lung alveolar cells, cardiac myocytes, vascular endothelium, and the retina. ${ }^{5}$ In patients whose immune responses are overloaded and/or those with high viral loads, apoptosis of pneumocytes and endothelial cells activate platelets and coagulation factors, leading to increased inflammation and further damage to the pneumocytes, which can lead to increased oxygen demand, cytokine storm, acute respiratory distress syndrome, activation of the coagulation cascade, and thromboembolic disease. ${ }^{6}$ Fortunately, the usage of ACE inhibitors and angiotensin receptor blockers has not been associated with an increased risk of severe COVID-19 infection. ${ }^{7}$

The virus is primarily transmitted via respiratory droplets, ${ }^{8}$ but rare cases of vertical transmission from pregnant mothers to their children have also been reported. ${ }^{9}$ A systemic review found that $6.3 \%$ of babies born to mothers with COVID-19 tested positive for the infection at birth. ${ }^{10}$

\section{Mortality and Morbidity}

Approximately $20 \%$ of infected patients required hospitalization and $6 \%$ required critical care. ${ }^{11}$ The risk of fatality was up to $14 \%$ in those older than 75 years of age. ${ }^{6}$ In Sao Paulo, Brazil, there was an estimated $25 \%$ excess death in 2020 , of which $94 \%$ was attributed to SARS-CoV-2. ${ }^{12}$ COVID-19 was the third leading cause of death in the United States in 2020, with an age-related death rate of $0.83 \% .{ }^{8}$ Mortality rates were similar in southern India, but overall estimates of infection-fatality rates may have been limited by underreporting of deaths, especially in migrant populations that may have had worse baseline health. ${ }^{12,13}$

More than half of patients with COVID-19 have underlying comorbidities, including obesity, diabetes, hypertension, and cardiopulmonary diseases that may affect the severity and morbidity of the infection, the post-infection sequelae, and the risk of vaccine breakthrough. ${ }^{14}$ Diabetes is associated with chronic inflammation and endothelial dysfunction, and obesity is associated with upregulation of the interleukin (IL)-6 receptors and low-grade metaflammation. ${ }^{14}$ Endothelial dysfunction and hypoxia can lead to thrombin generation and fibrinolysis. ${ }^{15}$ Recently, there have been reports of increasing rates of opportunistic fungal infections like pulmonary aspergillosis and rhino-orbital-cerebral mucormycoses in COVID-19 patients in India (15-27\%), which may be due to underlying uncontrolled diabetes, impaired immune systems, corticosteroid use for the treatment of COVID19, and/or prolonged stays in intensive care units (ICUs). ${ }^{16,17}$ Some studies have also found an increased risk of infection and mortality from COVID-19 in patients with age-related macular degeneration (ARMD), which was hypothesized to be due to complement and coagulation cascade dysfunction, elderly patients' exposure as they obtained medical care, and other health comorbidities; however, the association between macular degeneration and morbidity may have been present even before the pandemic. $^{18,19}$

\section{Treatment}

Multiple potential treatment regimens have been investigated for the prevention and treatment of COVID-19, including monoclonal antibodies, glucocorticoids, and antivirals. The effectiveness may be partly dependent on the stage of the disease and the ability to decrease the inflammatory response.

Patients with non-severe COVID-19 infections who are not at high risk for severe disease are primarily treated with supportive care. In those at risk for progression, clinical trials have found that monoclonal antibodies including ritonavir, casirivimab, imdevimab, bamlanivimab, etesevimab, CT$\mathrm{P} 59$, and sotrovimab were associated with decreased rates of hospitalization compared to placebos; however, only casirivimab-imdevimab was found in a meta-analysis to have moderate certainty evidence for reducing hospitalizations. ${ }^{20}$ The combination of nirmatrelvir (SARS-CoV-2 main protease inhibitor) and ritonavir (HIV-1 protease inhibitor and CYP3A inhibitor) has gained emergency use approval by the Federal Drug Administration (Paxlovid, PF-07321332, Pfizer, New York, USA) for use in non-hospitalized patients with COVID-19 at high risk for progressing to severe illness to decrease the risk of hospitalization and death; in the phase $2 / 3$ randomized double blind study, there was an $89 \%$ reduction in hospitalization and deaths compared to placebo. ${ }^{21}$

In patients with severe or critical COVID-19, metaanalyses have found that glucocorticoids likely reduced the risk of death and mechanical ventilation and duration of hospitalization in admitted patients requiring oxygen support. ${ }^{22}$ There was moderate certainty that the antiviral remdesivir reduced the length of symptoms and duration of mechanical ventilation. ${ }^{22}$ IL-6 antagonists like tocilizumab were also associated with a lower risk of progression 
to mechanical ventilation or death, especially in those who were receiving corticosteroids. ${ }^{23}$ Janus kinase (JAK) inhibitors baricitinib and ruxolitinib were associated with lower rates of invasive mechanical ventilation and mortality compared to the standard of care. ${ }^{24}$

Some therapies were unfortunately not found to be effective in large clinical trials. A systemic review and meta-analysis of 12 clinical trials involving 8569 patients found that hydroxychloroquine and chloroquine had little to no effect on decreasing the risk of death or progression to mechanical ventilation in patients with COVID-19; indeed, these medications were associated with a threefold higher risk of any adverse events, though fortunately not serious adverse events like QT-interval prolongation, cardiac arrhythmia, or retinopathy. ${ }^{25}$ A meta-analysis found that it was highly uncertain that prophylactic ivermectin with or without iota-carrageenan reduced the risk of infection given the serious risk of bias in the clinical studies. ${ }^{26}$ In meta-analyses, antiviral antibodies and blood products like convalescent plasma and intravenous immunoglobulins were not associated with a reduction in mortality in those with severe disease. ${ }^{20,27,28}$ The benefits of other treatments like favipiravir, azithromycin, lopinavirritonavir, umifenovir are under investigation. ${ }^{22}$

\section{Thromboembolism and Prophylaxis}

Severe COVID-19 may be associated with acute coronary syndrome, and $1.3 \%$ may develop acute cerebrovascular disease. ${ }^{6,15}$ Approximately 14\% develop venous thromboembolisms ( $46 \%$ of those in the ICU compared to $23 \%$ in non-ICU patients). ${ }^{6}$ For the prevention of thromboembolic complications in non-critically ill patients hospitalized with COVID-19, a randomized control trial of 2219 patients found that therapeutic-dose anticoagulation with heparin increased the probability of survival to hospital discharge and decreased the need for ICU-level organ support when compared to usual-care thromboprophylaxis, such as low or intermediate-dose thromboprophylaxis with enoxaparin, dalteparin, tinzaparin, fondaparinux, or heparin; in contrast, there was no difference in the risk of thromboembolism based on the type of thromboprophylaxis for critically ill patients in the ICU. ${ }^{29,30}$

\section{Long-Term Systemic Effects of COVID}

While most patients, especially those with milder symptoms, recovered from COVID-19, some may develop persistent or new symptoms four or more weeks after their infection. A large meta-analysis found that $80 \%$ of patients developed one or more long-term symptoms, most commonly fatigue $(58 \%)$, headache $(44 \%)$, attention disorder $(27 \%)$, dyspnea $(24 \%)$, and anosmia $(21 \%) .{ }^{31}$ Other symptoms included myalgias, hair loss $(25 \%)$, digestive disorders $(12 \%)$, sleep issues, fever, dizziness, rash, mood changes, autoimmune conditions, and post-traumatic stress disorder. $^{8,31}$ A meta-analysis found that $39 \%$ of recovered patients had altered pulmonary diffusion capacity, ${ }^{11}$ and $34 \%$ had abnormal chest X-rays or computerized tomography (CT) scans. ${ }^{31}$ Those who had severe disease with high inflammatory indicators were more likely to develop pulmonary fibrosis. ${ }^{11}$ Former COVID-19 patients who were referred for rehabilitation services had poorer physical function than cancer patients; ${ }^{32}$ therefore, patients who recover from COVID-19 may need long-term follow-up care.

\section{Ocular Manifestations in Adults and Children}

Ophthalmic manifestations of SARS-CoV-2 are not common, affecting approximately $6-12 \%$ of COVID-19 patients. $^{2,33-35}$ The ocular symptoms can precede systemic symptoms by 3 hours to 5 days in $13 \%$ of patients. ${ }^{2}$ Frequent hand-eye contact and more severe systemic illness may be more likely to be associated with ocular findings. 36

\section{Anterior Segment Findings}

In systemic reviews, the most common signs and symptoms were conjunctivitis $(86 \%)$, ocular pain $(31-34 \%)$, dry eyes $(33 \%)$, discharge $(19 \%)$, and redness $(11 \%))^{2,33,37}$ The presence of COVID-19 in the tear film and anterior surface varied from $0 \%$ to $13 \%,{ }^{2}$ and approximately 4 $24 \%$ had viral ribonucleic acid (RNA) detectable in conjunctival swabs. ${ }^{2,33,37}$ Eye protection and frequent hand hygiene may be considered to minimize the risks of transmission. $^{38}$ For those who develop conjunctivitis, some authors have proposed using topical eye drops with broad-spectrum antiviral activity, like povidone iodine and sodium hypochlorite. ${ }^{39}$ Fortunately, most ocular symptoms were mild and resolved, with or without treatment. ${ }^{2,36}$

In the ICU, ocular complications like exposure keratopathy and corneal abrasions may occur in patients who are on ventilators or respiratory masks; efforts to ensure adequate lubrication and closure of the eyelids may help prevent these ocular surface issues. In the outpatient setting, quarantine dry eye syndrome may occur as people 
socially distance indoors and use technology more frequently; artificial tears and rest may help alleviate these symptoms. ${ }^{40}$

\section{Posterior Segment Findings}

Histopathologic specimens from the retinas of deceased patients with COVID-19 have found presumed SARS$\mathrm{CoV}-2$ viral particles in the ganglion cell layer, inner plexiform, inner nuclear, outer plexiform, outer nuclear layer, retinal pigment epithelium, and choroid. ${ }^{41}$ A literature review found that COVID-19 was associated with an 8.86-fold risk of retinal vascular microvasculopathy, including retinal hemorrhages, cotton wool spots, infarcts at the internal plexus, and thinner ganglion cell layer and inner nuclear layer. ${ }^{42}$ More severe systemic disease was associated with lower vascular density on OCT angiography (OCTA). ${ }^{43}$ Other potential posterior segment findings included possible hyperreflective lesions in the inner and outer retina and vitreous, ${ }^{1,2,44}$ uveitis-like multifocal evanescent white dot syndrome, ${ }^{45}$ acute macular neuroretinopathy, ${ }^{46}$ central retinal vein occlusion, ${ }^{47}$ intraretinal hemorrhages, ${ }^{48}$ and inflammatory multifocal chorioretinitis with Adie's pupil. ${ }^{49}$ These changes may reflect the underlying systemic hypercoagulable, ischemic, inflammatory, and/or hypertensive state of the patient or direct invasion of the virus. $1,2,44,46-48,50$

\section{Neuro-Ophthalmic Findings}

Up to $21 \%$ of patients with COVID-19 may have systemic neurologic signs, and $0.4 \%$ have cranial nerve impairment. ${ }^{15}$ Potential neuro-ophthalmic associations with COVID-19 include optic neuritis and myelitis, ischemic optic neuropathy, Guillain-Barre syndrome, Miller-Fisher syndrome, and cranial neuropathies like CNIII or CNVI palsies. ${ }^{51}$ These neurologic conditions may be related to hypoxia, ischemia, and/or inflammation. ${ }^{52}$ An immunologic response to peripheral nerve antigens may result in demyelination and nerve injury. ${ }^{15}$ A systemic review found a higher prevalence of Guillain-Barre syndrome in patients with COVID-19 than in the general population ( $0.15 \%$ vs $0.02 \%$, respectively), which was believed to be due to an immune-mediated response rather than a direct viral infection. ${ }^{53}$

\section{Ocular Findings in Children}

Children who are hospitalized with COVID-19 may be more likely than adults to have ocular manifestations (up to $23 \%$ ), including conjunctival discharge $(55 \%)$ and conjunctival congestion $(10 \%) ;{ }^{54}$ posterior segment findings include retinal hemorrhages $(9 \%)$, cotton wool spots $(7 \%)$, dilated veins $(28 \%)$, and tortuous vessels $(13 \%)$. ${ }^{55}$ In Bergamo, Italy, there was a 30 -fold increase in the number of children with severe Kawasaki-like disease compared to before the pandemic; the majority of these children had IgG and/or IgM to SARS-CoV-2, were older, had a higher rate of cardiac involvement, and had more severe disease. ${ }^{56} \mathrm{~A}$ case series of 15 COVID-19+ newborns in Mexico found high rates of chemosis and hemorrhagic conjunctivitis $(73 \%)$, ciliary injection $(53 \%)$, and hyaline secretions $(100 \%) .{ }^{55}$ In contrast, a study of 165 newborns exposed to SARS-CoV-2 in Brazil found that only 6 patients had polymerase chain reaction (PCR)confirmed COVID-19, and none had vascular abnormalities that were attributed to SARS-CoV-2. ${ }^{9}$ Some ocular findings in children with COVID-19 may be related to their underlying systemic health.

\section{Ophthalmic Clinical Services and Effects of Delayed Care}

Even before the pandemic, there were barriers to healthcare, with delayed care being associated with worse visual acuity outcomes. Patients who were older than 80 years old, non-white, and had lower incomes were more likely to be lost to follow-up. ${ }^{57}$ Delays of 3 months or more in antivascular endothelial growth factor (VEGF) treatment were associated with persistently decreased visual acuities in patients with neovascular ARMD, even with resumption of treatment and return to baseline retinal thicknesses. ${ }^{58}$

During the initial phases of the pandemic, many patients delayed seeking medical care, even for lifethreatening conditions like myocardial infarctions and cerebrovascular accidents. ${ }^{59,60}$ Hospitals and outpatient clinics limited services to concentrate on combating COVID-19 and decreasing viral transmission. Adaptations to ophthalmic clinical and emergency services included referral for virtual consultations when possible, minimizing wait times, isolating care teams, and using triaging algorithms. ${ }^{1,61,62}$ The American Academy of Ophthalmology, Centers for Disease Control, World Health Organization, and American Society of Retina Surgeons released clinical guidelines. ${ }^{1}$ At the National Center for Global Health and Medicine in Japan, flowcharts were created for hospitalists to determine whether to consult ophthalmology for hospitalized patients with COVID-19, based on the consciousness levels and ocular 
signs and symptoms. ${ }^{63}$ At the University of Michigan, a risk stratification tool was used to determine patients' glaucoma severity and risk of glaucoma progression using the Sight Outcomes Research Collaborative (SOURCE) ophthalmology electronic health data repository data to determine when the patients should be evaluated in clinic. $^{62}$ The Royal College of Ophthalmologists released guidelines to prioritize urgent and emergent ophthalmic care, including maintaining anti-VEGF therapy every 8 weeks for patients with neoavascular age-related macular degeneration and consider deferring injections for most patients with macular edema from diabetes mellitus or vein occlusions. ${ }^{64}$

\section{Ocular Emergencies and Effects of Delayed Care}

Ophthalmic emergency room visits and outpatient office visits decreased by approximately $25-81 \%$ in MarchApril 2020 compared to the previous year. ${ }^{61,65-67}$ In Italy, there was a $59 \%$ reduction in patient visits to the ophthalmic emergency department, and in Spain, there was a $65 \%$ decrease during the lockdown. ${ }^{68}$

Patients who did present to the emergency departments unfortunately had more delayed presentations, more trauma, more severe injuries like ruptured globes, lacerations, and orbital fractures, more at-home injuries, and were less likely to have health insurance. ${ }^{61,65,68}$ The greater proportion of severe injuries may have been associated with increased intimate partner violence and home improvement projects as people quarantined. ${ }^{67}$

For the anterior segment, patients who presented during the pandemic in India were more likely to have more severe corneal ulcers $(22 \%$ vs $60 \%, P<0.001)$, more frank perforations ( $4 \%$ vs $18 \%, P=0.002$ ), poorer treatment successes $(51 \%$ vs $71 \%, P=0.007)$, and a higher proportion of anatomical failure $(24 \%$ vs $2 \%, P<0.001)$ compared to patients who presented in the prior year. ${ }^{69}$ In Ireland, there was a $37 \%$ reduction in the number of infectious keratitis during the pandemic compared to the prior 2 years, but the patients who did present had less improvement in their visual recovery compared to prior years (0.45 $\log$ MAR vs $0.26-0.67 \log$ MAR, $P=0.04) .^{70}$ There was also an increased incidence of alcohol-based hand sanitizer injury to the corneal surface in children. ${ }^{71}$ While there was a $81 \%$ reduction in glaucoma outpatient visits in India, there was a $62 \%$ increase in the proportion of glaucoma emergencies. ${ }^{72}$
Some studies found a $55-80 \%$ decrease in retinal detachment repairs during lockdown periods in $2020,{ }^{73,74}$ but others found a 39\% increase for urgent surgeries, such as retinal tears, retinal detachments, and trauma. ${ }^{75}$ One study of 82 patients with rhegmatogenous retinal detachments at Wills Eye Hospital found that the patients in 2020 tended to present later than in prior years $(20 \%$ within a day vs $37 \%$, respectively, $P=0.005$ ), were less likely to have their maculas still attached $(24 \%$ vs $50 \%, P=0.001)$, had worse presenting visual acuities (1.00logMAR vs $0.48 \log$ MAR, $P=0.008$ ), and were more likely to have grade $\mathrm{C}$ proliferative vitreoretinopathy (13\% PVR vs $5 \%$, $P=0.03){ }^{60}$ Similarly, Awad et al found that patients who presented with retinal detachments after lockdown measures were implemented in the United Kingdom were more likely to have PVR ( $10 \%$ vs $24 \%, P=0.047){ }^{76}$ The initially decreased rates of retinal detachment surgeries may therefore reflect delays in care.

Several studies have found worse outcomes in patients whose anti-VEGF treatments were delayed. There was an approximately $10-75 \%$ decrease in intravitreal injections in 2020 compared to 2019 , with at least $42 \%$ directly attributed to the pandemic. ${ }^{77}$ In the United Kingdom, Stone et al found that in 981 eyes of 858 patients, patients with wet ARMD lost a mean of 5.18 ETDRS letters, vein occlusion patients 5.15 letters, and diabetics 2.37 letters; even after resuming anti-VEGF treatments, less patients with wet ARMD and vein occlusions returned to baseline than diabetics $\left(75-77 \%\right.$ vs $90 \%$, respectively). ${ }^{64}$ Fortunately, $27 \%$ of patients with wet ARMD did not develop recurrent subretinal fluid despite an average 13 weeks delay. ${ }^{64}$ Rush et al similarly found that their patients in Texas with an average of 12 weeks delay in treatment had worse visual acuities that did not recover back to baseline, despite resuming anti-VEGF treatments for at least 6 months. $^{78}$ In a study of 109 patients in Turkey, $58 \%$ of whom had delayed care (on average 98 days), patients had worse visual acuity and more activity on follow-up OCTs. ${ }^{79}$ In Jordan, a mean delay of 61 days was associated with worse visual acuities. ${ }^{80}$ In Turkey, 7 of 106 patients (7\%) developed submacular hemorrhages during the restriction period. ${ }^{81}$ While the central macular thickness improved to pre-restriction levels, the vision at the last follow-up did not significantly improve; linear logistic regression found that the time interval between the injections was correlated with the visual acuity. ${ }^{81}$ In Cleveland, Song et al found that patients with an average of 5 weeks delay also had vision loss, more pronounced in 
those with diabetes and/or vein occlusions than neovascular ARMD. ${ }^{82}$ In Italy, patients who were evaluated in retina clinics during or immediately after the quarantine period had worse visual acuities compared to prior visits; in multiple regression analyses, the time period between visits showed the greatest association with changes in visual acuity. ${ }^{83}$ In Miami, patients whose intravitreal injections were delayed by an average of 3 weeks were more likely to have at least a 3 letter visual loss. ${ }^{77}$ Indeed, a study in Minnesota found that even a 2-week delay was associated with worse central subfield thicknesses and worse visual acuities, with a difference of approximately 5 letters. ${ }^{84} \mathrm{~A}$ theoretical model found that patients could lose vision as quickly as 1 letter per month of deferred treatment, outpacing the potential $0.17-0.56$ letter visual gain per month that patients could gain with antiVEGF therapy for ARMD. ${ }^{85}$ While some patients may therefore be able to tolerate a short delay in treatment, it is important to try to restart treatment as quickly as feasible for patients once lockdown restrictions have lifted in order to improve the visual potential. Importantly, masks did not appear to increase the risk of ocular infections after intravitreal injections. A large multicenter retrospective study of over 500,000 intravitreal injections found that the rates of presumed endophthalmitis were similar when there was universal masking of the physician, ancillary staff, and patients (with or without tape across the top of the mask) compared to no face masks by the physician or patient; however, the rates of culture-positive endophthalmitis were lower in the universal masking group. ${ }^{86}$

\section{Uveitis and Immunosuppressive Therapy}

The initiation or continuation of immunosuppressive therapy for non-infectious uveitis has been debated. Approximately $6-15 \%$ of patients with non-infectious uveitis may contract COVID-19. ${ }^{87}$ In a survey of 150 uveitis patients, $20 \%$ of respondents had stopped their medications during the pandemic, and 34\% reporting worsening of their ocular condition. ${ }^{88}$ In a national claims-based database search of 29,869 patients with non-infectious uveitis, uveitis patients had higher rates of COVID-19 infections (5.7\% VS 4.2\%, $P<0.001$ ), hospitalizations $(1.2 \%$ vs $0.6 \%, P<0.001)$, and death $(0.3 \%$ vs $0.1 \%, P<0.001)$, but this association was attributed to the patients' comorbidities and medication use rather than the uveitis itself. After adjusting for demographics and comorbidities like autoimmune disease, cardiovascular disease, diabetes, and chronic kidney disease, systemic steroids were associated with an increased risk of COVID-19 infection (hazards ratio for 1.19, 95\% CI, 1.18-1.20, $P<0.001$ ), hospitalization, and death. ${ }^{87}$ While tumor necrosis-alpha (TNF- $\alpha$ ) inhibitors like infliximab were also associated with an increased risk of infection, there have been conflicting reports in the literature as to whether they might have a protective effect by mitigating the inflammatory response. ${ }^{87,89}$

The consensus opinion from a survey of uveitis specialists from the International Uveitis Study Group, the International Ocular Inflammation Society, the Uveitis Society of India, and the Foster Ocular Immunology Society was to try local therapy rather than systemic steroids when possible and to avoid starting systemic steroids or immunosuppressive therapy (IMT) in those with suspected or confirmed COVID-19. ${ }^{90}$ Patients already on immunomodulatory therapy, biologics, or non-steroidal anti-inflammatory drugs without signs of COVID-19 may continue their medications but should practice hand hygiene, social distancing, and wear masks. ${ }^{90}$ In a study of 59 patients on biologic agents like infliximab, adalimumab, and rituximab, 15\% tested positive for COVID-19 but none exhibited symptoms; the biologics were held temporarily until the viruses cleared, and 2 patients $(22 \%)$ developed flares that were successfully treated by resuming their biologic agents. Immunocompromised patients may therefore be asymptomatic carriers, and their IMTs may be held temporarily if they contract COVD-19. ${ }^{89}$

\section{Teleophthalmology}

Teleophthalmology has been used for years to remotely screen for diabetic retinopathy, retinopathy of prematurity (ROP), macular degeneration, glaucoma, and anterior segment pathologies. The pandemic highlighted the importance of developing the infrastructure for telehealth options.

Approximately $22-73 \%$ of ophthalmic emergency department visits before the pandemic were estimated to be for non-urgent issues. At Moorfields Eye Hospital, approximately $57 \%$ of cases presenting to the emergency departments were referred for virtual consultations, with $21 \%$ being referred to the emergency department or a subspecialty service. ${ }^{61}$ In Pittsburgh, only $8 \%$ of those with telehealth visits required urgent, in-person evaluations; the majority of telehealth visits were for routine postoperative visits, followed by conjunctivitis. ${ }^{91}$ 
Ways to expand telehealth include developing secure, Health Insurance Portability and Accountability Act (HIPAA)-compliant programs, increasing access to the internet and technological devices, improving patient education device usage, quickly and accurately reviewing screenings, and ensuring appropriate and timely in-person examinations and treatments. ${ }^{92}$ Home OCTs and smart phone apps measuring vision, contrast sensitivity, ocular alignment, subretinal fluid, and metamorphopsia may be used for at-home monitoring and earlier detection of disease. ${ }^{93-95}$ Fundus photographs, slit lamp photography, laser flare photometry, fluorescein angiography, OCTs, and OCTAs can be obtained with minimally trained staff, be remotely operated, or be self-operated..$^{93,96}$

Artificial intelligence (AI) or a remote screener can help evaluate the images. Current AI screenings have sensitivities ranging from $75-95 \%$ for diabetic retinopathy, $87-100 \%$ for macular degeneration, $95 \%$ for ROP, 67 $93 \%$ for glaucoma, and $70 \%$ for cataracts. ${ }^{92,93}$ Those with unreadable images (approximately 5-20\%) or abnormalities detected remotely can then be referred in for in-person evaluations. ${ }^{93}$ In areas where there may be a dearth of subspecialties like uveitis, hybrid visits may be considered where a general ophthalmologist can perform the exam and imaging, then consult a uveitis specialist electronically. ${ }^{96}$ As the pandemic continues, hybrid visits where patients get imaging in person and counseling virtually may help ease patient concerns while still maintaining ophthalmic care.

\section{Vaccine Effectivity and Potential Side Effects \\ Vaccine Effectiveness}

Over 7 billion doses of COVID-19 vaccines have already been administered. ${ }^{3}$ In a multicenter study of 64,400 hospital or emergency department (ED) and urgent care (UC) visits between January 1-June 22, 2021, the messenger RNA (mRNA)-based BNT162b2 (Comirnaty, PfizerBioNTech, United States) and mRNA-1273 vaccine (Moderna, United States) vaccines were found to be $89 \%$ effective against laboratory-confirmed SARS-CoV-2 infections leading to hospitalizations, $90 \%$ effective against ICU admissions, and $91 \%$ against $\mathrm{ED} / \mathrm{UC}$ visits. ${ }^{97}$ Comparing the mRNA-based vaccines in July 2021, when the delta variant was more prevalent in Minnesota, the mRNA-1273 vaccine (Moderna) was estimated to be slightly more effective than the BNT162b2 (Pfizer) vaccine in preventing infections $(86 \%$ vs $76 \% P<0.001)$, hospitalizations ( $92 \%$ vs $85 \%, P<0.001)$, and ICU admissions $(93 \%$ vs $87 \%, P=0.0005)$, but both were highly effective in preventing deaths ( $0 \%$ in both groups). ${ }^{98}$ The BNT162b2 vaccine was found to be $91-95 \%$ effective in children over 5 years old. ${ }^{99}$

Most of the vaccines had lower efficacies when administered as a single dose or against the COVID-19 variants. The single-dose adenovirus-based Ad26.COV2 (Johnson \& Johnson/Janssen, Netherlands) was 54\% effective in preventing hospitalization and $68 \%$ effective against ED/UC visits. ${ }^{97}$ One dose of the BNT162b2 (Pfizer) or the adenovirus vector-based ChAdOx1 nCoV19 (AstraZeneca, United Kingdom) vaccines was 49\% effective against the alpha variant and $31 \%$ against the delta variant. ${ }^{100}$ After two doses, however, the BNT162b2 vaccine was 94\% effective against the alpha and $88 \%$ against the delta while the ChAdOx $1 \mathrm{nCoV}-19$ vaccine (AstraZeneca) was $75 \%$ effective against the alpha and $67 \%$ against the delta variant. ${ }^{100}$ The spike proteinbased NVX-CoV2373 vaccine (Novavax, United States) was $51 \%$ effective against the beta variant. ${ }^{100}$ The twodose inactivated whole-virion SARS-CoV-2 vaccine CoronaVac (SinoVac Life Sciences, China) had an $84 \%$ efficacy in preventing symptomatic COVID-19 and 100\% against hospitalizations in Phase 3 clinical trials, ${ }^{101}$ and the two-dose heterologous recombinant adenovirus-based Gam-COVID-Vac (Sputnik V, Gamaleya National Centre for Epidemiology and Microbiology, Russia) had a reported $92 \%$ efficacy in phase 3 clinical trials. ${ }^{102}$

\section{Potential Systemic and Ocular Side Effects}

Common vaccine side effects include myalgia and pain at the injection site. ${ }^{8,98}$ Very rare but serious adverse effects include anaphylaxis, myocarditis, pericarditis, and "vaccine-induced thrombotic thrombocytopenia" (VITT). Patients with VITT have anti-platelet factor 4 (PF4) antibodies, which activate platelet aggregation, leading to systemic thromboses. In contrast to other hypercoagulable disorders, thrombosis in VITT can occur in atypical sites such as the splanchnic, adrenal, cerebral, and ophthalmic veins. ${ }^{103}$ The current criteria for diagnosis of VITT include COVID vaccination 4 to 42 days prior to symptom onset, any venous or arterial thrombosis, thrombocytopenia, positive anti-PF4 antibodies, and D-dimer levels greater than 4 times the upper limit of normal. ${ }^{104}$

The prothrombotic state induced by COVID vaccination may affect ocular and neurologic perfusion as well. Bayas 
et al reported a case of bilateral superior ophthalmic vein thrombosis, ischemic stroke, and thrombocytopenia after ChAdOx1 nCoV-19 (AstraZeneca) vaccination, and other unilateral case reports have also been published. ${ }^{105-107}$ Acute macular neuroretinopathy has been reported following the ChAdOx1 nCoV-19 vaccination, ${ }^{108,109}$ and CNVI palsy has been reported 2 days after BNT162b2 mRNA vaccine (Pfizer), which resolved after 3 months. ${ }^{110}$ Fan et al reported a case of branched retinal artery occlusion with features of paracentral acute middle maculopathy in a pediatric patient after BNT162b2 vaccination (personal communication). As of May 2021, the Royal College of Ophthalmology has issued a safety alert soliciting reports of vaccine-related retinal vascular occlusions due to an increased incidence of central venous sinus thrombosis and other anecdotal reports of RVO after vaccination. ${ }^{111}$ Given the rarity of such cases, further study is required before a causative link can be established.

Although rare, uveitis and graft rejections have previously been associated with vaccines against influenza, hepatitis $B$, tetanus, toxoid, and yellow fever. The proposed mechanisms of action include molecular mimicry, delayed hypersensitivity, or other immune responses. ${ }^{112-114}$ In a multi-center study of 23 eyes of 21 patients that developed an acute episode of uveitis after the BNT162b2 vaccine (Pfizer), 8 patients (38\%) had a known history of uveitis, the median time to onset was approximately 8 days, and most patients (86\%) had mild to moderate anterior uveitis that significantly improved with topical therapy or intravitreal steroids. ${ }^{115}$ The authors suggested that the vaccine may have triggered a type I interferon secretion, leading to an autoimmune reaction. ${ }^{115}$ There have also been case reports of acute corneal graft rejections occurring after vaccination, which may be due to allorecognition by the direct pathway or incitation of the antibody response; fortunately, most rejection episodes resolved with topical and/or systemic steroids. ${ }^{105,112,116-120}$ Physicians may therefore consider reviewing the potential signs of graft rejection with corneal transplant recipients in order to improve earlier detection and treatment.

Systemically, the vaccines have fortunately not been associated with a significantly high risk of transplant rejection. A large study of 741 solid organ transplant recipients (STOR) who received either the BNT162b2 (Pfizer) or mRNA-1273 (Moderna) vaccines found that only 1 person developed a graft rejection after the second dose. ${ }^{121}$ In patients with graft-versus-host disease, there was no evidence that the vaccines triggered or worsened the disease. ${ }^{122}$ SOTR and hematopoietic stem cell patients, who are typically on immunosuppressants and are at higher risk for developing infections, are encouraged to receive the vaccines and boosters, even if they mount less of an antibody response. ${ }^{122,123}$

\section{Impact on Ophthalmic Business, Research, and Education}

While the primary focus of physicians during the pandemic has been on providing quality medical care in a safe environment, medical practices have also had to deal with the financial burdens of the pandemic. Clinical and surgical volumes were decreased as patients socially distanced, personal protective equipment was in short supply, and medical resources were diverted to caring for hospitalized patients.

In March 2020, the United States Congress passed the Coronavirus Aid, Relief and Economic Security (CARES) Act, which provided for accelerated Medicare payments for providers, tax relief for medical practices, no cost testing for COVID-19, and payroll tax credits. The Small Business Administration's Paycheck Protection Program (PPP) offered zero-fee loans as part of the efforts to help small business severely impacted by the pandemic with payroll support. Approximately $88 \%$ of physicians responded to a survey asking if they had applied for federal financial aid, and 96\% had successfully obtained funding. ${ }^{124}$ Physicians are reporting a rebound in clinical volume, but there may still be a lag. ${ }^{124}$ In terms of medicolegal liabilities, the CARES Act provided some legal protection to health-care workers when they were volunteering, but similar legal protections were not universal and did not apply to employed health-care workers. ${ }^{125}$ Additional liability protection should be considered as physicians manage the complications of delayed care and continued clinical and surgical restrictions. While the Centers for Medicare and Medicaid Services (CMS) paid for telemedicine visits during the pandemic, the additional growth of teleophthalmology may be partly dependent on how those telehealth visits will be reimbursed in the future.

As hospital systems try to recoup loss revenue, financial support from academic institutions for research may be shifted elsewhere. Some research staff were furloughed during the pandemic, some experimental cell lines and animal models that took months or years to establish were adversely affected, and grants were delayed. Basic science research projects were halted, and clinical data was significantly affected by the pandemic restrictions and delays in care. Scientists have advocated for additional 
government research support as they try to recover and restart their research. ${ }^{126}$

In medical education, universities and hospitals cancelled clinical electives and away rotations, leaving future physicians with decreased clinical exposure and confidence about their preparedness, which can lead to missed diagnoses or over-referrals. ${ }^{127}$ Students interested in ophthalmology, especially those without an associated ophthalmology department in their medical school, may have had greater difficulty obtaining clinical exposure, research, mentorship, and letters of recommendation when applying for residency. ${ }^{127}$ The advent of teleophthalmology, online computer-based learning, videoconferencing, and virtual simulators have been used to try to increase medical student exposure to ophthalmology. ${ }^{127}$

\section{Discussion}

COVID-19 has caused significant complications on the systemic and ocular health of patients and strained the global healthcare system. The SARS-CoV-2 virus is highly infectious, and patients with diabetes, immunosuppression, cardiopulmonary diseases, and metabolic syndromes may be more susceptible. Preventative methods include social distancing, masks, and vaccinations. For those who do contract the virus, systemic treatment options include monoclonal antibodies and antiviral agents in those at risk for severe disease, therapeutic dose heparin in non-critically ill hospitalized patients, JAK inhibitors to decrease the risk of invasive mechanical ventilation and mortality, IL-6 antagonists in patients with severe or critical COVID-19, and steroids in hospitalized patients requiring oxygen support. Most patients recover from the respiratory illness, but some may develop persistent pulmonary disease and fatigue. While the SARS-CoV-2 virus and the vaccines may both be associated with inflammatory or neurologic issues, the benefits of vaccinations far outweigh the rare potential complications.

In the eyes, the SARS-CoV-2 virus has been found in the conjunctiva, tear film, and retina, and it can cause conjunctivitis, microvascular disease, and cranial neuropathies. Fortunately, most ocular issues resolved, with or without treatment.

During the initial wave of the pandemic and quarantine period, there was a significant reduction in the clinical and surgical volumes. Teleophthalmology helped to minimize the risks of in-person evaluations for nonurgent issues. Those who did present to the emergency departments tended to have more severe disease and worse visual outcomes, and patients who had delayed their routine outpatient ophthalmic care had difficulty returning to their baseline statuses even after restarting treatments. Resumption of ophthalmic treatments as soon as possible may therefore help decrease the risk of vision loss. Continued financial support of ophthalmic businesses and research will help preserve the availability of ophthalmologists and prevent the loss of scientific advancement. Supplemental virtual didactics may help prepare the next generation of physicians and ophthalmologists.

Ongoing research has shed greater insight into how the virus affects the human body, potential treatment options, and the effects of delayed care for ophthalmic diseases. The lessons and insights gained from researchers around the world will hopefully help improve patient care as the pandemic continues.

\section{Disclosure}

Dr Harry W Flynn Jr reports grants from NIH Grant, grants from Research to Prevent Blindness, during the conduct of the study. None of the authors have any other conflicts of interest to disclose.

\section{References}

1. Leung EH, Flynn HW, Gayer S, et al. Clinical and perioperative management in ophthalmology during the COVID-19 pandemic. Int Ophthalmol Clin. 2020;60(3):141-158. doi:10.1097/ IIO.0000000000000310

2. Inomata $\mathrm{T}$, Kitazawa $\mathrm{K}$, Kuno $\mathrm{T}$, et al. Clinical and prodromal ocular symptoms in Coronavirus disease: a systematic review and meta-analysis. Invest Ophthalmol Vis Sci. 2020;61(10):29. doi:10.1167/iovs.61.10.29

3. WHO COVID-19 dashboard. Available from: https://who.sprinklr. com/. Accessed April 15, 2020.

4. Krause PR, Fleming TR, Longini IM, et al. SARS-CoV-2 variants and vaccines. $N$ Engl $J$ Med. 2021;385(2):179-186. doi:10.1056/ NEJMsr2105280

5. Bikdeli B, Madhavan MV, Jimenez D, et al. COVID-19 and thrombotic or thromboembolic disease: implications for prevention, antithrombotic therapy, and follow-up: JACC state-of-the-art review. J Am Coll Cardiol. 2020;75(23):2950-2973. doi:10.1016/j.jacc.2020.04.031

6. Leentjens J, van Haaps TF, Wessels PF, Schutgens REG, Middeldorp S. COVID-19-associated coagulopathy and antithrombotic agents-lessons after 1 year. Lancet Haematol. 2021;8(7):e524-e533. doi:10.1016/S2352-3026(21)00105-8

7. Flacco ME, Acuti Martellucci C, Bravi F, et al. Treatment with ACE inhibitors or ARBs and risk of severe/lethal COVID-19: a meta-analysis. Heart Br Card Soc. 2020;106(19):1519-1524. doi:10.1136/heartjnl-2020-317336

8. CDC. Coronavirus disease 2019 (COVID-19). Centers for Disease Control and Prevention; 2020. Available from: Available from: https://www.cdc.gov/coronavirus/2019-ncov/hcp/guidance-riskassesment-hcp.html. Accessed April 10, 2020. 
9. Kiappe OP, Santos da Cruz NF, Rosa PAC, Arrais L, Bueno de Moraes NS. Ocular assessments of a series of newborns gestationally exposed to maternal COVID-19 infection. JAMA Ophthalmol. 2021;139(7):777-780. doi:10.1001/jamaophthalmol.2021.1088

10. Bwire GM, Njiro BJ, Mwakawanga DL, Sabas D, Sunguya BF. Possible vertical transmission and antibodies against SARS-CoV2 among infants born to mothers with COVID-19: a living systematic review. J Med Virol. 2021;93(3):1361-1369. doi:10.1002/ jmv. 26622

11. Torres-Castro R, Vasconcello-Castillo L, Alsina-Restoy X, et al. Respiratory function in patients post-infection by COVID-19: a systematic review and meta-analysis. Pulmonology. 2021;27 (4):328-337. doi:10.1016/j.pulmoe.2020.10.013

12. Fernandes GA, Azevedo E, Silva G, et al. Excess mortality by specific causes of deaths in the city of São Paulo, Brazil, during the COVID-19 pandemic. PLoS One. 2021;16(6):e252238. doi:10.1371/journal.pone. 0252238

13. Cai R, Novosad P, Tandel V, Asher S, Malani A. Representative estimates of COVID-19 infection fatality rates from four locations in India: cross-sectional study. BMJ Open. 2021;11(10): e050920. doi:10.1136/bmjopen-2021-050920

14. Steenblock C, Schwarz PEH, Ludwig B, et al. COVID-19 and metabolic disease: mechanisms and clinical management. Lancet Diabetes Endocrinol. 2021;9:786-798. doi:10.1016/S22138587(21)00244-8

15. Cagnazzo F, Arquizan C, Derraz I, et al. Neurological manifestations of patients infected with the SARS-CoV-2: a systematic review of the literature. $J$ Neurol. 2021;268(8):2656-2665. doi:10.1007/s00415-020-10285-9

16. Raut A, Huy NT. Rising incidence of mucormycosis in patients with COVID-19: another challenge for India amidst the second wave? Lancet Respir Med. 2021;9(8):e77. doi:10.1016/S22132600(21)00265-4

17. Dilek A, Ozaras R, Ozkaya S, Sunbul M, Sen EI, Leblebicioglu H. COVID-19-associated mucormycosis: case report and systematic review. Travel Med Infect Dis. 2021;44:102148. doi:10.1016/j.tmaid.2021.102148

18. Ramlall V, Thangaraj PM, Meydan C, et al. Immune complement and coagulation dysfunction in adverse outcomes of SARS-CoV2 infection. Nat Med. 2020;26(10):1609-1615. doi:10.1038/ s41591-020-1021-2

19. Tuuminen R, Hecht I, Kanclerz P. Age-related macular degeneration and mortality in SARS-CoV-2-infected patients. Acta Ophthalmol (Copenh). 2020. doi:10.1111/aos.14685

20. Siemieniuk RA, Bartoszko JJ, Díaz Martinez JP, et al. Antibody and cellular therapies for treatment of covid-19: a living systematic review and network meta-analysis. BMJ. 2021;374:n2231. doi:10.1136/bmj.n2231

21. Pfizer.Pfizer's Novel COVID-19 Oral Antiviral Treatment Candidate Reduced Risk of Hospitalization or Death by $89 \%$ in Interim Analysis of Phase 2/3 EPIC-HR Study. 5 Nov 2021. Available from: https://www.pfizer.com/news/press-release/pressrelease-detail/pfizers-novel-covid-19-oral-antiviral-treatment-can didate. Accessed 19 Dec 2021.

22. Siemieniuk RA, Bartoszko JJ, Ge L, et al. Drug treatments for covid-19: living systematic review and network meta-analysis. BMJ. 2020;370:m2980. doi:10.1136/bmj.m2980

23. Shankar-Hari M, Vale CL, Mateo GM, et al. Association between administration of IL- 6 antagonists and mortality among patients hospitalized for COVID-19: a meta-analysis. JAMA. 2021;326 (6):499-518. doi:10.1001/jama.2021.11330

24. Ngamprasertchai T, Kajeekul R, Sivakorn C, et al. Efficacy and safety of immunomodulators in patients with COVID-19: a systematic review and network meta-analysis of randomized controlled trials. Infect Dis Ther. 2021. doi:10.1007/s40121-02100545-0
25. Singh B, Ryan H, Kredo T, Chaplin M, Fletcher T. Chloroquine or hydroxychloroquine for prevention and treatment of COVID-19. Cochrane Database Syst Rev. 2021;2:CD013587. doi:10.1002/ 14651858.CD013587.pub2

26. Bartoszko JJ, Siemieniuk RAC, Kum E, et al. Prophylaxis against covid-19: living systematic review and network meta-analysis. BMJ. 2021;373:n949. doi:10.1136/bmj.n949

27. Estcourt LJ, Turgeon AF, Turgeon AF, et al. Effect of convalescent plasma on organ support-free days in critically ill patients with COVID-19: a randomized clinical trial. JAMA. 2021;326:1690-1702. doi:10.1001/jama.2021.18178

28. Janiaud P, Axfors C, Schmitt AM, et al. Association of convalescent plasma treatment with clinical outcomes in patients with COVID-19: a systematic review and meta-analysis. JAMA. 2021;325(12):1185-1195. doi:10.1001/jama.2021.2747

29. ATTACC Investigators, ACTIV-4a Investigators, REMAP-CAP Investigators. Therapeutic anticoagulation with heparin in noncritically ill patients with Covid-19. $N$ Engl J Med. 2021;385 (9):790-802. doi:10.1056/NEJMoa2105911.

30. REMAP-CAP, ACTIV-4a, and ATTACC Investigators. Therapeutic anticoagulation with heparin in critically ill patients with Covid-19. $N$ Engl $J$ Med. 2021. doi:10.1056/ NEJMoa2 103417

31. Lopez-Leon S, Wegman-Ostrosky T, Perelman C, et al. More than 50 long-term effects of COVID-19: a systematic review and meta-analysis. Res Sq. 2021;rs.3.rs-266574. doi:10.21203/rs.3. rs-266574/v1.

32. Rogers-Brown JS, Wanga V, Okoro C. Outcomes among patients referred to outpatient rehabilitation clinics after COVID-19 diagnosis - United States, January 2020March 2021. MMWR Morb Mortal Wkly Rep. 2021;70. doi:10.15585/mmwr.mm7027a2

33. Aggarwal K, Agarwal A, Jaiswal N, et al. Ocular surface manifestations of coronavirus disease 2019 (COVID-19): a systematic review and meta-analysis. PLoS One. 2020;15(11):e241661. doi:10.1371/journal.pone.0241661

34. Feng Y, Park J, Zhou Y, Armenti ST, Musch DC, Mian SI. Ocular manifestations of hospitalized COVID-19 patients in a tertiary care academic medical center in the United States: a cross-sectional study. Clin Ophthalmol Auckl NZ. 2021;15:1551-1556. doi:10.2147/OPTH.S301040

35. Ulhaq ZS, Soraya GV. The prevalence of ophthalmic manifestations in COVID-19 and the diagnostic value of ocular tissue/fluid. Graefes Arch Clin Exp Ophthalmol. 2020;1-2. doi:10.1007/ s00417-020-04695-8

36. Chen L, Deng C, Chen X, et al. Ocular manifestations and clinical characteristics of 535 cases of COVID-19 in Wuhan, China: a cross-sectional study. Acta Ophthalmol (Copenh). 2020;98(8): e951-e959. doi:10.1111/aos.14472

37. Arora R, Goel R, Kumar S, et al. Evaluation of SARS-CoV-2 in tears of patients with moderate to severe COVID-19. Ophthalmology. 2021;128(4):494-503. doi:10.1016/j.ophtha.2020.08.029

38. Napoli PE, Nioi M, d'Aloja E, Fossarello M. The ocular surface and the Coronavirus disease 2019: does a dual "ocular route" exist? J Clin Med. 2020;9(5):E1269. doi:10.3390/ jcm9051269

39. Napoli PE, Mangoni L, Gentile P, Braghiroli M, Fossarello M. A panel of broad-spectrum antivirals in topical ophthalmic medications from the drug repurposing approach during and after the Coronavirus disease 2019 era. J Clin Med. 2020;9(8):E2441. doi: $10.3390 / \mathrm{jcm} 9082441$

40. Napoli PE, Nioi M, Fossarello M. The "quarantine dry eye": the lockdown for Coronavirus disease 2019 and its implications for ocular surface health. Risk Manag Healthc Policy. 2021;14:1629-1636. doi:10.2147/RMHP.S277067 
41. Araujo-Silva CA, Marcos AAA, Marinho PM, et al. Presumed SARS-CoV-2 viral particles in the human retina of patients with COVID-19. JAMA Ophthalmol. 2021;139:1015. doi:10.1001/ jamaophthalmol.2021.2795

42. Teo KY, Invernizzi A, Staurenghi G, Cheung CMG. COVID-19 related retinal micro-vasculopathy - a review of current evidence: COVID-19 related retinal micro-vasculopathy. Am J Ophthalmol. 2021;S0002-9394(21)00476-1. doi:10.1016/j.ajo.2021.09.019

43. Zapata MÁ, Banderas García S, Sánchez-Moltalvá A, et al. Retinal microvascular abnormalities in patients after COVID-19 depending on disease severity. Br J Ophthalmol. 2020;bjophthalmol-2020-317953. doi:10.1136/bjophthalmol-2020-317953.

44. Costa ÍF, Bonifácio LP, Bellissimo-Rodrigues F, et al. Ocular findings among patients surviving COVID-19. Sci Rep. 2021;11:11085. doi:10.1038/s41598-021-90482-2

45. De Salvo G, Meduri A, Vaz-Pereira S, Spencer D. An uncommon cold of the retina. Surv Ophthalmol. 2021. doi:10.1016/j. survophthal.2021.08.006

46. Preti RC, Zacharias LC, Cunha LP, Monteiro MLR. Acute macular neuroretinopathy as the presenting manifestation of COVID-19 infection. Retin Cases Brief Rep. 2021;Publish Ahead of Print. doi:10.1097/ICB.0000000000001050

47. Ullah I, Sohail A, Shah MU, et al. Central Retinal Vein Occlusion in patients with COVID-19 infection: a systematic review. Ann Med Surg. 2021;71:102898. doi:10.1016/j.amsu.2021.102898

48. Monferrer-Adsuara C, Castro-Navarro V, González-Girón N, et al. A case of bilateral unusual retinal hemorrhages in a COVID-19 patient. Eur J Ophthalmol. 2020;1120672120984381. doi:10.1177/ 1120672120984381.

49. Ortiz-Seller A, Martínez costa L, Hernández-Pons A, Valls Pascual E, Solves Alemany A, Albert-Fort M. Ophthalmic and neuro-ophthalmic manifestations of Coronavirus disease 2019 (COVID-19). Ocul Immunol Inflamm. 2020;28(8):1285-1289. doi:10.1080/09273948.2020.1817497

50. Yahalomi T, Pikkel J, Arnon R, Pessach Y. Central retinal vein occlusion in a young healthy COVID-19 patient: a case report. Am J Ophthalmol Case Rep. 2020;20:100992. doi:10.1016/j. ajoc. 2020.100992

51. Tisdale AK, Chwalisz BK. Neuro-ophthalmic manifestations of coronavirus disease 19. Curr Opin Ophthalmol. 2020;31 (6):489-494. doi:10.1097/ICU.0000000000000707

52. Gold DM, Galetta SL. Neuro-ophthalmologic complications of coronavirus disease 2019 (COVID-19). Neurosci Lett. 2021;742:135531. doi:10.1016/j.neulet.2020.135531

53. Palaiodimou L, Stefanou M-I, Katsanos AH, et al. Prevalence, clinical characteristics and outcomes of Guillain-Barré syndrome spectrum associated with COVID-19: a systematic review and meta-analysis. Eur J Neurol. 2021;28(10):3517-3529. doi:10.1111/ene.14860

54. Ma N, Li P, Wang X, et al. Ocular manifestations and clinical characteristics of children with laboratory-confirmed COVID-19 in Wuhan, China. JAMA Ophthalmol. 2020;138(10):1079-1086. doi:10.1001/jamaophthalmol.2020.3690

55. Pérez-Chimal LG, Cuevas GG, Di-Luciano A, Chamartín P, Amadeo G, Martínez-Castellanos MA. Ophthalmic manifestations associated with SARS-CoV-2 in newborn infants: a preliminary report. $J$ AAPOS. 2021;25(2):102-104. doi:10.1016/j.jaapos.2020.11.007

56. Verdoni L, Mazza A, Gervasoni A, et al. An outbreak of severe Kawasaki-like disease at the Italian epicentre of the SARS-CoV-2 epidemic: an observational cohort study. Lancet. 2020;395 (10239):1771-1778. doi:10.1016/S0140-6736(20)31103-X

57. Obeid A, Gao X, Ali FS, et al. Loss to follow-up among patients with neovascular age-related macular degeneration who received intravitreal anti-vascular endothelial growth factor injections. JAMA Ophthalmol. 2018;136(11):1251-1259. doi:10.1001/ jamaophthalmol.2018.3578
58. Greenlee TE, Wang VY, Kang H, et al. Consequences of lapses in treatment with vascular endothelial growth factor inhibitors in neovascular age-related macular degeneration in routine clinical practice. Retina Phila Pa. 2021;41(3):581-587. doi:10.1097/ IAE.0000000000002888

59. Tam -C-CF, Cheung K-S, Lam S, et al. Impact of Coronavirus disease 2019 (COVID-19) outbreak on ST-segment-elevation myocardial infarction care in Hong Kong, China. Circ Cardiovasc Qual Outcomes. 2020;13(4). doi:10.1161/CIRCOUTCOMES.120.006631

60. Patel LG, Peck T, Starr MR, et al. Clinical presentation of rhegmatogenous retinal detachment during the COVID-19 pandemic: a historical cohort study. Ophthalmology. 2021;128(5):686-692. doi:10.1016/j.ophtha.2020.10.009

61. Wickham L, Hay G, Hamilton R, et al. The impact of COVID policies on acute ophthalmology services-experiences from Moorfields Eye Hospital NHS Foundation Trust. Eye. 2020;34 (7):1189-1192. doi:10.1038/s41433-020-0957-2

62. Bommakanti NK, Zhou Y, Ehrlich JR, et al. Application of the sight outcomes research collaborative ophthalmology data repository for triaging patients with glaucoma and clinic appointments during pandemics such as COVID-19. JAMA Ophthalmol. 2020;138(9):974-980. doi:10.1001/jamaophthalmol.2020.2974

63. Yashiro $S$, Ueta $T$, Kutsuna $S$, Okamoto $T$, Nagahara $M$, Ohmagari N. Using flowchart for ophthalmic consultations in hospitalized patients with COVID-19. Glob Health Med. 2020;2 (6):395-397. doi:10.35772/ghm.2020.01091

64. Stone LG, Grinton ME, Talks JS, Cui J, Sun H, Lu X. Delayed follow-up of medical retina patients due to COVID-19: impact on disease activity and visual acuity. Graefes Arch Clin Exp Ophthalmol. 2021;259:1-8. doi:10.1007/s00417-021-05174-4

65. Wu C, Patel SN, Jenkins TL, Obeid A, Ho AC, Yonekawa Y. Ocular trauma during COVID-19 stay-at-home orders: a comparative cohort study. Curr Opin Ophthalmol. 2020;31 (5):423-426. doi:10.1097/ICU.0000000000000687

66. Cavuoto KM, Vanner EA, Osigian CJ. Trends in pediatric ocular trauma presenting to an ophthalmology-specific emergency department during the COVID-19 pandemic. J AAPOS. 2021;25 (3):170-172. doi:10.1016/j.jaapos.2021.01.004

67. Halawa OA, Friedman DS, Roldan AM, Zebardast N. Changing trends in ocular trauma during the COVID-19 pandemic in the USA. $\quad B r \quad J$ Ophthalmol. 2021;bjophthalmol-2021-319627. doi:10.1136/bjophthalmol-2021-319627

68. Franzolin E, Casati S, Albertini O, et al. Impact of Covid-19 pandemic on Ophthalmic Emergency Department in an Italian tertiary eye centre. Eur J Ophthalmol. 2021;1120672121998223. doi: $10.1177 / 1120672121998223$.

69. Christy JS, Mathews P, Rhagavan A, et al. Impact of COVID-19 pandemic on infectious keratitis outcomes: a retrospective multicenter study in tertiary eye hospitals of South India. Cornea. 2021;Publish Ahead of Print. doi:10.1097/ICO.0000000000002829

70. Power B, Donnelly A, Murphy C, Fulcher T, Power W. Presentation of infectious keratitis to ED during COVID-19 lockdown. J Ophthalmol. 2021;2021:5514055. doi:10.1155/ 2021/5514055

71. Martin GC, Le Roux G, Guindolet D, et al. Pediatric eye injuries by hydroalcoholic gel in the context of the Coronavirus disease 2019 pandemic. JAMA Ophthalmol. 2021;139(3):348-351. doi:10.1001/jamaophthalmol.2020.6346

72. Rajendrababu S, Durai I, Mani I, Ramasamy KS, Shukla AG, Robin AL. Urgent and emergent glaucoma care during the COVID-19 pandemic: an analysis at a tertiary care hospital in South India. Indian $J$ Ophthalmol. 2021;69(8):2215-2221. doi:10.4103/ijo.IJO_635_21

73. Shams F, El-Abiary M, Goudie C, Yorston D. Effects of lockdown on retinal detachment incidence in Scotland. Eye Lond Engl. 2021;35(4):1279-1280. doi:10.1038/s41433-020-1029-3 
74. Schranz M, Georgopoulos M, Sacu S, et al. Incidence and surgical care of retinal detachment during the first SARS-CoV-2 lockdown period at a tertiary referral center in Austria. PLoS One. 2021;16(3):e0248010. doi:10.1371/journal.pone.0248010

75. Moon JY, Miller JB, Katz R, et al. The impact of the COVID-19 pandemic on ophthalmic care at an eye-specific emergency department in an outbreak hotspot. Clin Ophthalmol. 2020;14:4155-4163. doi:10.2147/OPTH.S285223

76. Awad M, Poostchi A, Orr G, Kumudhan D, Zaman A, Wilde C. Delayed presentation and increased prevalence of proliferative vitreoretinopathy for primary rhegmatogenous retinal detachments presenting during the COVID-19 pandemic lockdown. Eye Lond Engl. 2021;35(4):1282-1283. doi:10.1038/s41433020-1056-0

77. Ashkenazy N, Goduni L, Smiddy WE. Short-term effects of COVID-19-related deferral of intravitreal injection visits. Clin Ophthalmol Auckl NZ. 2021;15:413-417. doi:10.2147/OPTH. S296345

78. Rush RB, Rush SW. Outcomes in patients resuming intravitreal anti-VEGF therapy following treatment delay during the COVID-19 pandemic. Retina Phila Pa. 2021;41:2456-2461. doi:10.1097/IAE.0000000000003276

79. Sevik MO, Aykut A, Özkan G, Dericioğlu V, Şahin Ö. The effect of COVID-19 pandemic restrictions on neovascular AMD patients treated with treat-and-extend protocol. Int Ophthalmol. 2021;41:2951-2961. doi:10.1007/s10792-021-01854-6

80. Elfalah M, AlRyalat SA, Toro MD, et al. Delayed intravitreal anti-VEGF therapy for patients during the COVID-19 lockdown: an ethical endeavor. Clin Ophthalmol Auckl NZ. 2021;15:661-669. doi:10.2147/OPTH.S289068

81. Yeter DY, Dursun D, Bozali E, Ozec AV, Erdogan H. Effects of the COVID-19 pandemic on neovascular age-related macular degeneration and response to delayed anti-VEGF treatment. J Fr Ophtalmol. 2021;44(3):299-306. doi:10.1016/j.jfo.2021.02.001

82. Song W, Singh RP, Rachitskaya AV. The effect of delay in care among patients requiring intravitreal injections. Ophthalmol Retina. 2021;5:SS2468. doi:10.1016/j.oret.2020.12.020

83. Borrelli E, Grosso D, Vella G, et al. Short-term outcomes of patients with neovascular exudative AMD: the effect of COVID-19 pandemic. Graefes Arch Clin Exp Ophthalmol Albrecht Von Graefes Arch Klin Exp Ophthalmol. 2020;258 (12):2621-2628. doi:10.1007/s00417-020-04955-7

84. Naravane AV, Mundae R, Zhou Y, et al. Short term visual and structural outcomes of anti-vascular endothelial growth factor (anti-VEGF) treatment delay during the first COVID-19 wave: a pilot study. PLoS One. 2021;16(2):e0247161. doi:10.1371/journal.pone.0247161

85. Mollan SP, Fu DJ, Chuo C-Y, et al. Predicting the immediate impact of national lockdown on neovascular age-related macular degeneration and associated visual morbidity: an INSIGHT Health Data Research Hub for Eye Health report. $\mathrm{Br}$ $J$ Ophthalmol. 2021;bjophthalmol-2021-319383. doi:10.1136/ bjophthalmol-2021-319383.

86. Patel SN, Tang PH, Storey PP, et al. The influence of universal face mask use on endophthalmitis risk after intravitreal antivascular endothelial growth factor injections. Ophthalmology. 2021;128:1620-1626. doi:10.1016/j.ophtha.2021.05.010

87. Miller DC, Sun Y, Chen EM, Arnold BF, Acharya NR. The association between non-infectious uveitis and COVID-19 outcomes: an analysis of United States claims-based data. Ophthalmology. 2021;S0161-6420(21)00751-X. doi:10.1016/j. ophtha.2021.10.007

88. Abd Elmohsen MN, Youssef MM, Mamdouh Esmat S, Teleb DA, Tolba DA. Consequences of COVID-19 on uveitis patients from their own perspective: a questionnaire-based study. Ocul Immunol Inflamm. 2021;1-6. doi:10.1080/09273948.2021.1964029
89. AlBloushi AF, Alfawaz AM, Abu El Asrar AM. Implications of COVID-19 infection on patients with uveitis under biologic treatment. Br J Ophthalmol. 2021;bjophthalmol-2020-318577. doi:10.1136/bjophthalmol-2020-318577

90. Agrawal R, Testi I, Lee CS, et al. Evolving consensus for immunomodulatory therapy in non-infectious uveitis during the COVID-19 pandemic. Br J Ophthalmol. 2021;105(5):639-647. doi:10.1136/bjophthalmol-2020-316776

91. Kalra G, Williams AM, Commiskey PW, et al. Incorporating video visits into ophthalmology practice: a retrospective analysis and patient survey to assess initial experiences and patient acceptability at an academic eye center. Ophthalmol Ther. 2020;9 (3):549-562. doi:10.1007/s40123-020-00269-3

92. Walsh L, Hong SC, Chalakkal RJ, Ogbuehi KC. A systematic review of current teleophthalmology services in New Zealand compared to the four comparable countries of the United Kingdom, Australia, United States of America (USA) and Canada. Clin Ophthalmol Auckl NZ. 2021;15:4015-4027. doi: 10.2147/OPTH.S294428

93. Sommer AC, Blumenthal EZ. Telemedicine in ophthalmology in view of the emerging COVID-19 outbreak. Graefes Arch Clin Exp Ophthalmol. 2020;1-12. doi:10.1007/s00417-020-04879-2

94. Islam M, Sansome S, Das R, et al. Smartphone-based remote monitoring of vision in macular disease enables early detection of worsening pathology and need for intravitreal therapy. BMJ Health Care Inform. 2021;28(1):e100310. doi:10.1136/bmjhci-2020-100310

95. Khurana RN, Hoang C, Khanani AM, Steklov N, Singerman LJ. A smart mobile application to monitor visual function in diabetic retinopathy and age-related macular degeneration: the CLEAR study. Am J Ophthalmol. 2021;227:222-230. doi:10.1016/j. ajo.2021.03.033

96. Brill D, Papaliodis G. Uveitis specialists harnessing disruptive technology during the COVID-19 pandemic and beyond. Semin Ophthalmol. 2021;36(4):296-303. doi:10.1080/ 08820538.2021.1896753

97. Thompson MG, Stenehjem E, Grannis S, et al. Effectiveness of Covid-19 vaccines in ambulatory and inpatient care settings. $N$ Engl J Med. 2021;385(15):1355-1371. doi:10.1056/ NEJMoa2110362

98. Puranik A, Lenehan PJ, Silvert E, et al. Comparison of two highly-effective mRNA vaccines for COVID-19 during periods of Alpha and Delta variant prevalence. medRxiv. 2021;2021.08.06.21261707. doi:10.1101/2021.08.06.21261707.

99. Walter EB, Talaat KR, Sabharwal C, et al. Evaluation of the BNT162b2 Covid-19 vaccine in children 5 to 11 years of age. N Engl J Med. 2021;null. doi:10.1056/NEJMoa2116298.

100. Lopez Bernal J, Andrews N, Gower C, et al. Effectiveness of Covid-19 vaccines against the B.1.617.2 (Delta) variant. $N \quad$ Engl J Med. 2021;385(7):585-594. doi:10.1056/ NEJMoa2108891

101. Tanriover MD, Doğanay HL, Akova M, et al. Efficacy and safety of an inactivated whole-virion SARS-CoV-2 vaccine (CoronaVac): interim results of a double-blind, randomised, placebo-controlled, phase 3 trial in Turkey. Lancet Lond Engl. 2021;398(10296):213-222. doi:10.1016/S0140-6736(21) 01429-X

102. Logunov DY, Dolzhikova IV, Shcheblyakov DV, et al. Safety and efficacy of an rAd26 and rAd5 vector-based heterologous prime-boost COVID-19 vaccine: an interim analysis of a randomised controlled phase 3 trial in Russia. Lancet. 2021;397(10275):671-681. doi:10.1016/S0140-6736(21) 00234-8

103. Cines DB, Bussel JB. SARS-CoV-2 vaccine-induced immune thrombotic thrombocytopenia. $N$ Engl $J$ Med. 2021;384 (23):2254-2256. doi:10.1056/NEJMe2106315 
104. Bussel J, Connors J, Cines D, et al. Thrombosis with thrombocytopenia syndrome - hematology.org. thrombosis with thrombocytopenia syndrome (also termed vaccine-induced thrombotic thrombocytopenia). Available from: https://www.hematology. org:443/covid-19/vaccine-induced-immune-thromboticthrombocytopenia. Accessed August 21, 2021.

105. Bayas A, Menacher M, Christ M, Behrens L, Rank A, Naumann M. Bilateral superior ophthalmic vein thrombosis, ischaemic stroke, and immune thrombocytopenia after ChAdOx1 nCoV-19 vaccination. Lancet Lond Engl. 2021;397 (10285):e11. doi:10.1016/S0140-6736(21)00872-2

106. Panovska-Stavridis I, Pivkova-Veljanovska A, Trajkova S, Lazarevska M, Grozdanova A, Filipche V. A rare case of superior ophthalmic vein thrombosis and thrombocytopenia following ChAdOx1 nCoV-19 vaccine against SARS-CoV-2. Mediterr $J$ Hematol Infect Dis. 2021;13(1):e2021048. doi:10.4084/ MJHID.2021.048

107. McDonnell T, Jain S, Jain S, McGlynn S, McGlynn S. Left inferior ophthalmic vein thrombosis due to VITT (Vaccine Induced Thrombotic Thrombocytopenia): a case report. QJM Mon J Assoc Physicians. 2021;hcab124. doi:10.1093/qjmed/hcab124

108. Bøhler AD, Strøm ME, Sandvig KU, Moe MC, Jørstad ØK. Acute macular neuroretinopathy following COVID-19 vaccination. Eye Lond Engl. 2021. doi:10.1038/s41433-02101610-1

109. Book BAJ, Schmidt B, Foerster AMH. Bilateral acute macular neuroretinopathy after vaccination against SARS-CoV-2. JAMA Ophthalmol. 2021;139(7):e212471. doi:10.1001/ jamaophthalmol.2021.2471

110. Reyes-Capo DP, Stevens SM, Cavuoto KM. Acute abducens nerve palsy following COVID-19 vaccination. $J$ AAPOS. 2021;25:SS1091-9. doi:10.1016/j.jaapos.2021.05.003

111. The Royal Coleege of Opthalmologists. Safety alert: retinal vein occlusions post COVID vaccination. The Royal College of Ophthalmologists; 2021. Available from: https://www.rcophth. ac.uk/2021/05/retinal-vein-occlusions-post-covid-vaccination/. Accessed August 21, 2021.

112. Wasser LM, Roditi E, Zadok D, Berkowitz L, Weill Y. Keratoplasty rejection after the BNT162b2 messenger RNA vaccine. Cornea. 2021;40(8):1070-1072. doi:10.1097/ ICO.0000000000002761

113. ElSheikh RH, Haseeb A, Eleiwa TK, Elhusseiny AM. Acute Uveitis following COVID-19 vaccination. Ocul Immunol Inflamm. 2021;1-3. doi:10.1080/09273948.2021.1962917

114. Goyal M, Murthy SI, Annum S. Bilateral Multifocal Choroiditis following COVID-19 vaccination. Ocul Immunol Inflamm. 2021;1-5. doi:10.1080/09273948.2021.1957123

115. Rabinovitch T, Ben-Arie-Weintrob Y, Hareuveni-Blum T, et al. Uveitis following the BNT162b2 mRNA vaccination against SARS-CoV-2 infection: a possible association. Retina Phila Pa. 2021;41:2462-2471. doi:10.1097/IAE.0000000000003277
116. de la Presa M, Govil A, Chamberlain WD, Holland EJ. Acute corneal epithelial rejection of LR-CLAL after SARS-CoV-2 vaccination. Cornea. 2021;Publish Ahead of Print. doi:10.1097/ ICO.0000000000002914

117. Yu S, Ritterband DC, Mehta I. Acute corneal transplant rejection after severe acute respiratory syndrome Coronavirus 2 mRNA-1273 vaccination. Cornea. 2021;Publish Ahead of Print. doi:10.1097/ICO.0000000000002886

118. Shah AP, Dzhaber D, Kenyon KR, Riaz KM, Ouano DP, Koo EH. Acute corneal transplant rejection after COVID-19 vaccination. Cornea. 2021. doi:10.1097/ICO.0000000000002878

119. Rallis KI, Ting DSJ, Said DG, Dua HS. Corneal graft rejection following COVID-19 vaccine. Eye Lond Engl. 2021. doi:10.1038/ s41433-021-01671-2

120. Phylactou M, Li J-PO, Larkin DFP. Characteristics of endothelial corneal transplant rejection following immunisation with SARS-CoV-2 messenger RNA vaccine. $\mathrm{Br} J$ Ophthalmol. 2021;105(7):893-896. doi:10.1136/bjophthalmol-2021-319338

121. Ou MT, Boyarsky BJ, Motter JD, et al. Safety and reactogenicity of 2 doses of SARS-CoV-2 vaccination in solid organ transplant recipients. Transplantation. 2021;105(10):2170-2174. doi:10.1097/TP.0000000000003780

122. Eberhardt CS, Balletto E, Cornberg M, Mikulska M. Coronavirus disease 2019 vaccination in transplant recipients. Curr Opin Infect Dis. 2021;34(4):275-287. doi:10.1097/ QCO.0000000000000739

123. FDA Commissioner. Coronavirus (COVID-19) update: FDA authorizes additional vaccine dose for certain immunocompromised individuals. FDA; 2021. Available from: https://www.fda. gov/news-events/press-announcements/coronavirus-covid-19update-fda-authorizes-additional-vaccine-dose-certainimmunocompromised. Accessed August 15, 2021.

124. Chen E, Parikh R. COVID-19 and ophthalmology: the pandemic's impact on private practices. American Academy of Ophthalmology; 2020. Available from: https://www.aao.org/eye net/article/pandemic-impact-on-private-practices. Accessed August 15, 2021.

125. Napoli PE, Nioi M, d'Aloja E, Fossarello M. Safety recommendations and medical liability in ocular surgery during the COVID-19 pandemic: an unsolved dilemma. $J$ Clin Med. 2020;9(5):E1403. doi:10.3390/jcm9051403

126. Jorkasky J, Davis M, Lee PP. Potential impact of COVID-19 disruptions on the next generation of vision scientists. JAMA Ophthalmol. 2021;139:896. doi:10.1001/jamaophthalmol.2021.1959

127. Wendt S, Abdullah Z, Barrett S, et al. A virtual COVID-19 ophthalmology rotation. Surv Ophthalmol. 2021;66(2):354-361. doi:10.1016/j.survophthal.2020.10.001
Clinical Ophthalmology

\section{Publish your work in this journal}

Clinical Ophthalmology is an international, peer-reviewed journal covering all subspecialties within ophthalmology. Key topics include: Optometry; Visual science; Pharmacology and drug therapy in eye diseases; Basic Sciences; Primary and Secondary eye care; Patient Safety and Quality of Care Improvements. This journal is indexed on PubMed
Central and CAS, and is the official journal of The Society of Clinical Ophthalmology (SCO). The manuscript management system is completely online and includes a very quick and fair peer-review system, which is all easy to use. Visit http://www.dovepress.com/ testimonials.php to read real quotes from published authors. 